\title{
SIMILAR SEQUENCES
}

BY E. T. BELL

1. Similarity. The interesting reciprocity between the Eulerian numbers $E$ and the pseudo-Eulerian numbers $E^{\prime}$ of Pascal,* which was noticed by Sinigallia, $\dagger$ is not a peculiarity of the particular sequences $E, E^{\prime}$, but is a simple instance of a general property of all sequences of numbers or of polynomials. According to this property, defined below and called similarity, any sequence is similar to an infinity of other sequences; there is no special reason for singling $E^{\prime}$ out of all the sequences similar to $E$.

Let $S \equiv S_{n}, S^{\prime} \equiv S_{n}^{\prime},(n=0,1, \cdots)$, be any sequences such that

$$
S_{n}=f_{n}\left(S_{0}^{\prime}, S_{1}^{\prime}, \cdots\right), \quad S_{n}^{\prime}=f_{n}\left(S_{0}, S_{1}, \cdots\right),
$$

for all integers $n \geqq 0$, so that the $n$th element $S_{n}$ of $S$ is the same function $f_{n}$ of elements of $S^{\prime}$ that $S_{n}^{\prime}$ is of elements of $S$. We shall say that $S, S^{\prime}$ are similar, and write $S \sim S^{\prime}$, and hence also $S^{\prime} \sim S$.

Let $S \sim S^{\prime}$, and let the relation, in symbolic or umbral notation, which enables us to express the elements of either of $S$, $S^{\prime}$ as functions of the elements of the other, be $R\left(S, S^{\prime}\right)=0$. If $R\left(S, S^{\prime}\right)$ is bilinear in the elements of $S, S^{\prime}$, we shall say that $S, S^{\prime}$ are bilinearly similar. It will be shown in $\$ 2$ that all the $S^{\prime}$ bilinearly similar to any given $S$ constitute a three-parameter family of sequences.

As an example, we state the difference equation which defines all $E^{\prime}$ bilinearly similar to Euler's $E$, using for $E$ the notation of Lucas. $\ddagger$

* E. Pascal, Rendiconti del R. Istituto Lombardo, (2), vol. 40 (1907), pp. 461-475.

$\dagger$ L. Sinigallia, Rendiconti di Palermo, vol. 24 (1907), pp. 222-228.

$\ddagger$ E. Lucas, Theorie des Nombres, Chap. 14. Sinigallia's $E_{2 n}$ is Lucas' $(-1)^{n} E_{2 n}$. It is a great convenience in using the symbolic method to fill any gaps that may occur in a given sequence with zeros, so that the index ranges over all integers $n \geqq 0$, adding a supplementary definition to give the positions of the interpolated zeros. Thus Sinigallia's $E_{2 n}$ and Pascal's $E_{2 n}{ }^{\prime},(n=0,1, \cdots)$, would be replaced by $E_{n}, E_{n}^{\prime},(n=0,1, \cdots)$, with the supplementary defini- 
The recurrence for the numbers $E$ may be written

$$
(E+2)^{n}+E^{n}-2=0 .
$$

Let $a, b, d$ be constants such that $a d-b^{2} \neq 0, a+b \neq 0$. Define $E^{\prime}$ by

$$
\begin{gathered}
E_{0}^{\prime}=-(b+d) /(a+b), \\
2 a\left(E^{\prime}+1\right)^{n+1}+b\left[\left(E^{\prime}+2\right)^{n+1}+E^{\prime n+1}\right]+2\left(b+2^{n} d\right)=0 .
\end{gathered}
$$

It may be shown $(\S 4)$ that

$$
\begin{aligned}
a E_{0} E_{0}^{\prime}+b\left(E_{0}+E_{0}^{\prime}\right)+d & =0, \\
a\left(E+E^{\prime}\right)^{n+1}+b\left(E^{n+1}+E^{\prime n+1}\right) & =0 .
\end{aligned}
$$

Hence $E \sim E^{\prime}$, and the similarity is bilinear. Taking $a=2$, $b=-1, d=0$, we get Pascal's $E^{\prime}$, and the equivalent of Sinigallia's result. When $S \sim S^{\prime}$, the elements of $S, S^{\prime}$ may be numbers, as in the above example, or polynomials ( $\$ 5$ ).

If the elements of $S, S^{\prime}$ are integers, their congruence properties $(\$ 6)$ may be readily investigated from the symbolic form of the similarity $S \sim S^{\prime}$ by an immediate application of known theorems concerning the residues of binomial coefficients. When further there are known arithmetical properties of one of $S, S^{\prime}$, as is the case for instance with $E$, these may be used to reduce the congruences found as just indicated.

2. Bilinear Similarities. Let $a, b, c, d$ be constants such that $a d-b c \neq 0$. In order that

$$
a x y+b x+c y+d=0,
$$

when solved for $x, y$ in turn, give $x$ the same function $\phi$ of $y$ that $y$ is of $x$, it is necessary and sufficient that $b=c$, and we have

tions $E_{2 n+1}=0, E_{2 n+1}^{\prime}=0,(n=0,1, \cdots)$. An account of the umbral calculus, which supplements that of Lucas, has been given in my Algebraic Arithmetic, 1927. In particular, the formal use of infinite processes has been justified by an abstract postulational treatment, which establishes an isomorphism with finite operations on one-rowed matrices; see also Transactions of this Society, vol. 25 (1923), pp. 135-154. We recall that if $\alpha$ is umbral, and $a, b$ are ordinaries different from zero,

Henceforth $n$ denotes an arbitrary integer $\geqq 0$.

$$
(a \alpha+b)^{0}=a^{0} \alpha^{0} b^{0}=\alpha_{0} ;(a \alpha+b)^{n}=\sum_{j=0}^{n}\left(\begin{array}{l}
n \\
j
\end{array}\right) a^{n-i} b^{j} \alpha_{n-j} .
$$




$$
\begin{gathered}
a x y+b(x+y)+d=0, \quad a d-b^{2} \neq 0, \\
-\phi(z)=(b z+d) /(a z+b), \quad x=\phi(y), \quad y=\phi(x) .
\end{gathered}
$$

Let $\xi, \eta$ be the respective umbrae of the sequences $\xi_{n}, \eta_{n}$, $(n=0,1, \cdots)$. The generators $x, y$ of $\xi, \eta$ are $x \equiv e^{\xi t}, y \equiv e^{\eta t}$, where $t$ is an indeterminate (ordinary). In order that $\xi, \eta$ be bilinearly similar it is necessary and sufficient that (1) hold, and that, if one of $\xi, \eta$ be given, say $\xi$, the successive elements $\eta_{0}, \eta_{1}, \cdots$, of $\eta$ be uniquely determined by (1) when $x, y$ are replaced by $e^{\xi t}, e^{\eta t}$,

$$
a e^{(\xi+\eta) t}+b\left(e^{\xi t}+e^{\eta t}\right)+d=0 .
$$

The last is an identity in $t$. Hence

$$
\begin{aligned}
a(\xi+\eta)^{0}+b\left(\xi^{0}+\eta^{0}\right)+d & =0, \\
a(\xi+\eta)^{n+1}+b\left(\xi^{n+1}+\eta^{n+1}\right) & =0, \quad(n \geqq 0) .
\end{aligned}
$$

The first of these gives

$$
\left(a \xi_{0}+b\right) \eta_{0}+\left(b \xi_{0}+d\right)=0,
$$

and the coefficient of $\eta_{n+1}$ in the second is $a \xi_{0}+b$. Hence a necessary and sufficient condition that $\eta_{0}, \eta_{1}, \cdots$ be determinate when $\xi$ is given is

$$
\xi_{0} \neq-b / a .
$$

When (3) is satisfied, the bilinear similarity $\xi \sim \eta$ is given by (2), and if $e^{\xi t} \equiv f(t)$, the generator $e^{\eta t}$ of any $\eta$ bilinearly similar to the given $\xi$ is, by (1),

$$
-e^{\eta t} \equiv[b f(t)+d] /[a f(t)+b], \quad a d-b^{2} \neq 0 .
$$

Examples are given in $\$ 4$.

3. General Similarity $\xi \sim \eta$. Let

$$
x \equiv x(t) \equiv e^{\xi t}, \quad y \equiv y(t) \equiv e^{\eta t}
$$

be the generators of any sequences $\xi, \eta$. Let $R(x, y)=0$ be the result of eliminating $t$ from $x \equiv x(t), y \equiv y(t)$, where $x(t), y(t)$ are ordinary (not symbolic) functions of $t$. Then $R\left(e^{\xi t}, e^{\eta t}\right)=0$ is an identity in $t$. Let the coefficient of $t^{n}(n \geqq 0)$ in the formal MacLaurin expansion of $R\left(e^{\xi t}, e^{\eta t}\right)$ be $R_{n}(\xi, \eta)$. Then

$$
R_{n}(\xi, \eta)=0 \text {. }
$$


Necessary and sufficient conditions for $\xi \sim \eta$ are $x=\psi(y)$, $y=\psi(x)$, and the non-vanishing of the coefficients of $\eta_{0}, \eta_{1}, \ldots$ (where $\xi$ is the given sequence) in (6) when $n=0,1, \cdots$, successively. The problem of determining all $\eta$ such that $\eta \sim \xi$, when $\xi$ is given, is reduced to that of finding the general formal power series solution of the functional equation $\psi(\psi(z))=z$. The linear fractional solutions were determined in $\$ 2$; for the rest, see $\S 7$.

4. Examples of Bilinear Similarities. We shall determine all $\eta$ in the respective cases

$$
(\xi, \eta)=\left(B, B^{\prime}\right),\left(R, R^{\prime}\right),\left(G, G^{\prime}\right),\left(E, E^{\prime}\right),
$$

where $B, R, G, E$ are the umbrae of the numbers of Bernoulli, Lucas, Genocchi, and Euler, such that $\xi \sim \eta$, and the similarity is bilinear. The notation of Lucas, (loc. cit. Chap. 14) is used for $B, R, G, E$, and $x, y, a, b, c, d$ are as in $\S 2$. Hence $a d-b^{2} \neq 0$. The given generators are

$$
\begin{aligned}
e^{B t} & =t /\left(e^{t}-1\right), & e^{R t} & =t e^{t} /\left(e^{2 t}-1\right), \\
e^{G t} & =2 t /\left(e^{t}+1\right), & e^{E t} & =2 e^{t} /\left(e^{t}+1\right) .
\end{aligned}
$$

Consider first $(\xi, \eta)=\left(B, B^{\prime}\right)$. Since $B_{0}=1$ (in Lucas' notation for the Bernoulli numbers), the condition (3) is here $a+b \neq 0$. By (4) the generator of $\eta \equiv B^{\prime}$ is

$$
-e^{B^{\prime} t}=\frac{b e^{B t}+d}{a e^{B t}+b}=\frac{b t+d\left(e^{t}-1\right)}{a t+b\left(e^{t}-1\right)},
$$

and we see in this case that the generator has a convergent MacLaurin expansion under the given condition $a+b \neq 0$. Equating coefficients of like powers of $t$ in

$$
-e^{B^{\prime} t}\left[a t+b\left(e^{t}-1\right)\right]=b t+d\left(e^{t}-1\right)
$$

and collecting results, we get, $(n \geqq 0)$,

$$
\begin{aligned}
& (n+2) a B^{\prime n+1}+b\left[\left(B^{\prime}+1\right)^{n+2}-B^{\prime n+2}\right]+d=0, \\
& B_{0}^{\prime}=-(b+d) /(a+b), \quad a d-b^{2} \neq 0, \quad a+b \neq 0 ; \\
& a\left(B+B^{\prime}\right)^{n+1}+b\left(B^{n+1}+B^{\prime n+1}\right)=0 .
\end{aligned}
$$

In the same way from the given generators for $R, G$ we find 
(8) $(n+2) a\left(R^{\prime}+1\right)^{n+1}+b\left[\left(R^{\prime}+2\right)^{n+2}-R^{\prime n+2}\right]$

$$
+(n+2) b+2^{n+2} d=0 \text {, }
$$

$R_{0}^{\prime}=-(b+2 d) /(a+2 b), \quad a d-b^{2} \neq 0, \quad a+2 b \neq 0 ;$

$a\left(R+R^{\prime}\right)^{n+1}+b\left(R^{n+1}+R^{\prime n+1}\right)=0$;

(9) $b\left[\left(G^{\prime}+1\right)^{n+2}+G^{n+2}\right]+2 a G^{\prime n+1}+d=0$,

$G_{0}^{\prime}=-d / b, \quad G_{1}^{\prime}=\left(a d-b^{2}\right) / b, \quad a d-b^{2} \neq 0, \quad b \neq 0 ;$

$a\left(G+G^{\prime}\right)^{n+1}+b\left(G^{n+1}+G^{n+1}\right)=0$.

The results for $E, E^{\prime}$ were given in $\$ 1$. The numbers $G$, in another notation, are the tangential coefficients of Pascal,* who defines in connection with them the pseudo-tangential coefficients $\beta^{\prime}$, by means of the generator (in effect)

$$
e^{\beta^{\prime} t} \equiv \frac{e^{2 t}-1}{4 e^{t}-e^{2 t}-1}
$$

The $\beta^{\prime \prime}$ such that $\beta^{\prime} \sim \beta^{\prime \prime}$ bilinearly are determined by the recurrence

$$
\begin{gathered}
(a-b)\left(\beta^{\prime \prime}+2\right)^{n+1}+4 b\left(\beta^{\prime \prime}+1\right)^{n+1}-(a+b) \beta^{\prime \prime n+1} \\
+2^{n+1}(b-d)+4 d=0, \\
\beta_{0}^{\prime}=-d / b, \quad a d-b^{2} \neq 0, \quad b \neq 0 .
\end{gathered}
$$

5. Similar Sequences of Polynomials. The simplest sequence of polynomials in $u$ associated with a given sequence of numbers $\alpha$ is the sequence $A(u, \alpha)$ of Appell polynomials constructed on $\alpha$,

$$
A_{n}(u, \alpha) \equiv(u+\alpha)^{n}, \quad(n=0,1, \cdots) . \dagger
$$

Let $z(t) \equiv e^{\alpha t}$ be the generator of $\alpha$, and $w(t)$ that of $A(u, \alpha)$. Then, obviously, $w(t)=e^{u t} z(t)$. To find the sequences of polynomials $A^{\prime}(u, \alpha)$ similar to $A(u, \alpha)$ we take

$$
x(t) \equiv w(t) \equiv e^{u t} z(t)
$$

* E. Pascal, Rendiconti di Palermo, vol. 23 (1907), pp. 358-366.

$\dagger$ For $\alpha=B, R, G, E$, the simplifications introduced by taking $(u+\alpha)^{n}$ as the $n$th polynomial, instead of some of the slightly different definitions in the literature, are so considerable that it might be worth while to adopt the Appell polynomials in preference to the others. 
in $\S \S 2,3$, and proceed as before to find the generator $y(t)$ of $A^{\prime}(u, \alpha)$, and hence the recurrence for $A^{\prime}(u, \alpha)$, from which the $A_{n}^{\prime}(u, \alpha)$ can be calculated successively. Since $A(0, \alpha)=\alpha$, these results include those for similar sequences of numbers.

As an example, we take the Euler polynomials $A_{n}(u, E)$ $\equiv(u+E)^{n}$, and find all $A_{n}^{\prime}(u, E)$ such that the similarity $A^{\prime} \sim A$, where $A \equiv A(u, E), A^{\prime} \equiv A^{\prime}(u, E)$, is bilinear. Proceeding as just outlined, we get

$$
\begin{gathered}
2 a\left(A^{\prime}+u+1\right)^{n+1}+b\left[\left(A^{\prime}+2\right)^{n+1}+A^{\prime n+1}\right] \\
+2 b\left[(u+1)^{n+1}+2^{n+1} d\right]=0, \\
A_{0}^{\prime}=-(2 b+d) /(a+b), \quad a+b \neq 0, \quad a d-b^{2} \neq 0 ; \\
a\left(A+A^{\prime}\right)^{n+1}+b\left(A^{n+1}+A^{\prime n+1}\right)=0 .
\end{gathered}
$$

For $a=2, b=-1, d=0, u=0$, this gives the similarity $E^{\prime} \sim E$ in $\S 1$.

6. Congruences. Returning to (2), (3), we assume that the $\xi_{n}, \eta_{n}(n \geqq 0)$ are integers.*

Let $p$ be prime. In (2) take $n+1=p$. Then

$$
\left(a \xi_{0}+b\right) \eta_{p}+\left(a \eta_{0}+b\right) \xi_{p} \equiv 0(\bmod p) .
$$

Applying the known result $\dagger$

$$
\left(\begin{array}{c}
p-j \\
h
\end{array}\right) \equiv\left(\begin{array}{c}
-j \\
h
\end{array}\right)(\bmod p), \quad(0 \leqq h<p-j<p),
$$

where, by definition,

$$
\begin{aligned}
& \left(\begin{array}{c}
-m \\
0
\end{array}\right)=1,\left(\begin{array}{c}
-m \\
n
\end{array}\right)=(-m)(-m-1) \cdots\left(\begin{array}{r}
-m-n+1) / n !, \\
(m>0, n>0),
\end{array}\right. \\
& \text { to (2), we get }
\end{aligned}
$$

$$
\begin{aligned}
& \left(a \xi_{0}+b\right) \eta_{p-j}+\left(a \eta_{0}+b\right) \xi_{p-j} \\
& +\sum_{h=1}^{p-j-1}\left(\begin{array}{c}
-j \\
h
\end{array}\right) \xi_{p-j-h} \eta_{h} \equiv 0(\bmod p) \\
& \quad(p>2, \quad 0<p-j<p) .
\end{aligned}
$$

* Sufficient conditions: $a, b, a d$ integers; $\xi_{n+1}(n \geqq 0)$ integers; $a \xi_{0}+b=1$. $\dagger$ Lucas, loc. cit., \$228. 
The remaining possibility, $n+1>p$, is covered by the following theorem.* Let the expression of $n+1$ in the scale of $p$ be

$$
\begin{gathered}
n+1=g_{s} p^{s}+g_{s-1} p^{s-1}+\cdots+g_{1} p+g_{0}, \\
0 \leqq g_{j}<p, \quad(j=0, \cdots, s-1), \quad g_{s} \neq 0, \quad g_{s}<p ;
\end{gathered}
$$

and let $\lambda, \mu$ be any umbrae. Then

$$
(\lambda+\mu)^{n+1} \equiv \prod_{j=0}^{s}\left(\lambda^{p^{j}}+\mu^{p^{j}}\right)^{g_{j}}(\bmod p),
$$

where all the indicated binomial expansions and subsequent multiplications are to be performed as in common algebra before exponents are lowered.

7. The Equation $\psi^{2}(x)=x$. This is a special case of Babbage's equation, on which there is an extensive literature. $\dagger$ Let $\chi^{-1}$ be the function inverse to $\chi$. Then, if $\psi=g$ is any solution of $\psi^{2}(x)=x$, Babbage's solution is $\psi=\chi^{-1} g \chi$. But this is not the general solution, as noted by Babbage himself. Ritt determines what solutions are given by Babbage's formula.

According to a result of Leau (1), p. 6, the solution in $\$ 2$ is the general solution under the conditions (a) uniformity in the entire plane, (b) no singularities other than poles or isolated essential singularities anywhere in the plane; the solution can have no essential singularities.

By the second part of a theorem of Pfeiffer (loc. cit.), if $\psi(x)$ is restricted to be analytic about the origin and to vanish there, the equation has either no solution, one, or two solutions, or an infinity of solutions.

\section{California Institute of Technology}

* Proved in my paper on Anharmonic polynomials, Transactions of this Society, vol. 34 (1922), p. 109.

$\dagger$ The following papers contain further information and references bearing on the special case (as well as the general). C. Babbage, Memoirs of the Analytical Society, 1813, Note 1, pp. 47-50. L. Leau (1), Bulletin de la Société Mathématique, vol. 26 (1898), pp. 5-9. L. Leau (2), Annales de la Faculté des Sciences de Toulouse, vol. 11 (1897), pp. E1-E110. J. R, Ritt, Annals of Mathematics, (2), vol. 17 (1915), pp. 113-122. A. A. Bennett, ibid., pp. 22-60. Several of the references in this paper relate to our problem. G. A. Pfeiffer, American Journal of Mathematics, vol. 37 (1915), p. 421. Babbage's solution is reproduced by H. Laurent, Traité d'A nalyse, vol. 6, 1890, pp. 243-244. 